\title{
A Numerical Method for Finding Multiple Co-Existing Solutions to Nonlinear Cooperative Systems
}

\author{
Xianjin Chen ${ }^{a}$, Jianxin Zhou ${ }^{a, *}$ and Xudong Yao ${ }^{b}$ \\ a Department of Mathematics, Texas A\&M University, College Station, TX 77843 \\ ${ }^{b}$ Department of Mathematics, University of Connecticut, Storrs, CT 06269
}

\begin{abstract}
In this paper, a local min-orthogonal method is developed to solve cooperative nonlinear elliptic systems for multiple co-existing solutions. A characterization of co-existing critical points of a dual functional is established and used as a mathematical justification for the method. The method is then implemented to numerically solve two coupled nonlinear Schrödinger equations which model spatial vector solitons propagating in a saturable bulk nonlinear medium for multiple co-existing solutions.
\end{abstract}

Keywords: Cooperative elliptic systems, min-orthogonal method, multiple solutions, coexisting states, vector solitons, coupled nonlinear Schrödinger equations

AMS(MOS) subject classifications: 35A40,35A15,58E05,58E30

\section{Introduction}

An understanding of the interaction of simple physical objects (e.g., scalar solitons) leading to the formation of more complex objects (e.g., vector solitons) is an ultimate goal of fundamental research in many key areas, such as condensed matter physics, dynamics of biomolecules, nonlinear optics, etc [13]. Recently rapidly developing techniques of atomic, molecular and optical physics [12] have opened a door to carry out more intrinsic investigation

\footnotetext{
${ }^{*}$ Corresponding author.

Email addresses: xjchen@math.tamu.edu (X. Chen), jzhou@math.tamu.edu (J. Zhou), xudong@math.uconn.edu (X. Yаo)
} 
on the complex and intriguing dynamics induced by the vector nature of numerous nonlinear phenomena. For instance, it has been experimentally observed $[11,13]$ that several optical beams generated by coherent sources can be combined to produce a multicomponent selftrapped beams, also called spatial vector solitons. Similar vector phenomena such as vortices have been observed [10, 12] in multicomponent Bose-Einstein condensates (BEC) and exploited to explain their dynamical properties.

Multicomponent solitons (vector solitons) are of typical vector phenomena which have recently witnessed a renewed interest because of more and more experimental realization in the aforementioned areas. In this paper, we shall study the interaction of two mutually incoherent $(2+1)$-dimensional optical beams $\left(E_{1}, E_{2}\right)$ propagating in a saturable bulk medium (e.g., photorefractive crystals) along $z$ direction, which leads to our model problem, i.e., a system of two coupled nonlinear Schrödinger (NLS) equations [8, 11],

$$
\left\{\begin{array}{l}
i \frac{\partial E_{1}}{\partial z}+\Delta E_{1}-\frac{E_{1}}{1+\left(\left|E_{1}\right|^{2}+\left|E_{2}\right|^{2}\right)}=0 \\
i \frac{\partial E_{2}}{\partial z}+\Delta E_{2}-\frac{E_{2}}{1+\left(\left|E_{1}\right|^{2}+\left|E_{2}\right|^{2}\right)}=0
\end{array}\right.
$$

where $\Delta=\frac{\partial^{2}}{\partial x^{2}}+\frac{\partial^{2}}{\partial y^{2}}$ is the transverse Laplacian. In study of stability/instability, pattern formation and other vector phenomena, co-existing standing solitary wave solutions (or solitons for short) to (1.1) of the form

$$
E_{1}=u(x, y) e^{-i \beta_{1} z}, E_{2}=v(x, y) e^{-i \beta_{2} z}, u \neq 0, v \neq 0
$$

are of particular interest, where $0<\beta_{1}, \beta_{2}<1$ are two propagation constants. After setting $\lambda_{i}=1-\beta_{i}(i=1,2), \gamma=\frac{\lambda_{2}}{\lambda_{1}}$ and rescaling the amplitudes, $\{u, v\} \rightarrow\left\{u / \sqrt{\lambda_{1}}, v / \sqrt{\lambda_{1}}\right\}$, and the coordinates, $\{x, y\} \rightarrow\left\{\sqrt{\lambda_{1}} x, \sqrt{\lambda_{1}} y\right\},(1.1)$ leads to a semilinear elliptic system [8, 11]

$$
\left\{\begin{array}{l}
-\Delta u(x, y)=-u(x, y)+\frac{I(x, y)}{1+\mu I(x, y)} u(x, y), \\
-\Delta v(x, y)=-\gamma v(x, y)+\frac{I(x, y)}{1+\mu I(x, y)} v(x, y),
\end{array}\right.
$$

where $\mu \equiv \lambda_{1}=1-\beta_{1} \in(0,1)$ is the saturation parameter (the limit $\mu \rightarrow 0$ corresponds to the Kerr medium), $I(x, y)=u^{2}(x, y)+v^{2}(x, y)$ is the total intensity, the nonlinear term $\frac{I(x, y)}{1+\mu I(x, y)}$ characterizes a saturable nonlinearity of the medium. In this paper, we aim to find multiple vector solitons to (1.1) or co-existing solutions to (1.3) in an ascending order of 
their instability index, i.e., the number of maximum linearly independent directions along which a small perturbation decreases the associated functional value.

Similar to system (1.3), several semilinear elliptic systems that are derived from the Schrödinger systems in other applications [8, 12, 23] share the same general form

$$
\left\{\begin{array}{l}
k_{1} \Delta u+\left(\Phi_{1}(x,|u(x)|,|v(x)|)-\alpha\right) u=0 \\
k_{2} \Delta v+\left(\Phi_{2}(x,|u(x)|,|v(x)|)-\beta\right) v=0
\end{array}\right.
$$

where $\alpha, \beta, k_{1}>0, k_{2}>0$ are some constants, $\Phi_{1}, \Phi_{2}$ are some nonlinear functions of $|u(x)|$ and $|v(x)|, x \in \mathbb{R}^{N}(N \geq 1)$. Note that $\left(\psi_{1}, \psi_{2}\right) \equiv\left(u(x) e^{i \alpha z}, v(x) e^{i \beta z}\right)$ represents a vector soliton in the original Schrödinger systems where $z$ can be a space or time variable. For instance, the study of the mutual interaction of two optical beams $\left(\psi_{1}, \psi_{2}\right)$ propagating in an inhomogeneous Kerr medium (e.g., a photonic crystal fiber) [23] gives rise to a coupled system of the form (1.4) with $k_{1}=k_{2}=1$ and

$$
\Phi_{1}=n_{a}+V(x)\left(\delta+\left|\psi_{1}\right|^{2}+\mu\left|\psi_{2}\right|^{2}\right), \Phi_{2}=n_{a}+V(x)\left(\delta+\left|\psi_{2}\right|^{2}+\mu\left|\psi_{1}\right|^{2}\right)
$$

and the study of two-component BEC leads to the well-known Gross-Pitaevskii (GP) system [12], which is also of the form (1.4) but with $k_{j}=\frac{\hbar}{2 m_{j}}(j=1,2)$ and

$$
\Phi_{1}=-\frac{1}{\hbar}\left[V_{1}+g_{1}\left|\psi_{1}\right|^{2}+g_{12}\left|\psi_{2}\right|^{2}-\Omega L_{z}\right], \Phi_{2}=-\frac{1}{\hbar}\left[V_{2}+g_{2}\left|\psi_{2}\right|^{2}+g_{12}\left|\psi_{1}\right|^{2}-\Omega L_{z}\right]
$$

When the nonlinear terms $\Phi_{1}, \Phi_{2}$ in (1.4) (see, e.g., equations (1.3), (1.5) or (1.6)) satisfy

$$
\begin{aligned}
& {\left[\Phi_{1}(x,|u(x)|,|v(x)|)-\alpha\right] u(x)=G_{u}(x, u(x), v(x)),} \\
& {\left[\Phi_{2}(x,|u(x)|,|v(x)|)-\beta\right] v(x)=G_{v}(x, u(x), v(x)),}
\end{aligned}
$$

for a function $G(x, u(x), v(x))$ which is $\mathcal{C}^{1}$ in the second and third variables, those problems are variational. Thus we consider a semilinear cooperative elliptic system of the form

$$
\begin{cases}-\Delta u(x)=G_{u}(x, u(x), v(x)), & x \in \Omega, u \in H^{1}(\Omega), \\ -\Delta v(x)=G_{v}(x, u(x), v(x)), & x \in \Omega, v \in H^{1}(\Omega), \\ u(x)=v(x)=0\left(\text { or } \frac{\partial u(x)}{\partial n}=\frac{\partial v(x)}{\partial n}=0\right), & x \in \partial \Omega,\end{cases}
$$

where $\Omega$ is a bounded open domain in $\mathbb{R}^{N}, n$ is the unit outer normal vector; $G: \bar{\Omega} \times \mathbb{R}^{2} \rightarrow \mathbb{R}$ is of class $\mathcal{C}^{1}$ in the second and third variables, satisfying the following hypotheses 
(A1) $|\nabla G(x, z)| \leq C\left(1+|z|^{p-1}\right), \quad \forall z \in \mathbb{R}^{2}$, a.e. $x \in \Omega$, for some constants $C>0$ and $2<p<\frac{2 N}{N-2}$ if $N \geq 3$ or $2<p<+\infty$ if $N=1,2$ (subcritical growth [30,33]),

(A2) $G_{u}(x, 0, v(x)) \equiv G_{v}(x, u(x), 0) \equiv 0$ (homogeneity),

(A3) $G(x, u, v)=o\left(|(u, v)|^{2}\right)$ or $G(x, u, v)<0$ when $|(u, v)| \rightarrow 0$,

where $\left(G_{u}, G_{v}\right)$ is the gradient of $G$ in the second and third variables $(u, v) \in \mathbb{R}^{2}$. Condition (A1) is imposed in order to apply the continuous embedding $W^{1, q}(\Omega) \subset L^{q^{*}}(\Omega)$, where $q^{*}=q N /(N-q)>q\left(q^{*}=\infty\right.$, if $\left.q \leq N\right)$. Here, $q=2$. Condition (A1) implies that a weak solution $\left(u^{*}, v^{*}\right)$ of $(1.7)$ is precisely a critical point of a dual $\mathcal{C}^{1}$-functional $J: H_{0}^{1}(\Omega) \times H_{0}^{1}(\Omega)\left(\right.$ or $H^{1}(\Omega) \times H^{1}(\Omega)$ with zero Neumann boundary conditions $) \rightarrow \mathbb{R}$ given by

$$
J(u, v)=\frac{1}{2} \int_{\Omega}\left(|\nabla u(x)|^{2}+|\nabla v(x)|^{2}\right) d x-\int_{\Omega} G(x, u(x), v(x)) d x,
$$

i.e., the Frechet derivative $J^{\prime}\left(u^{*}, v^{*}\right)=0$. It can be easily verified that $(0,0)$ is a local minimum of $J$ under (A2) and (A3). The existence of multiple nontrivial solutions to system (1.7) has been established for several subclasses [1,21,33,34,37]. Among those nontrivial solutions, solutions without a zero component are of particular interest in many applications $[8,12,23]$ since they represent so-called co-existing states. Thus, in this paper we are only interested in finding such co-existing states (solutions) to (1.7) and shall view all other solutions as trivial. Additionally, hypothesis (A2) implies that if one component (i.e., $u$ or $v$ ) is equal to zero, then system (1.7) reduces to a semilinear elliptic equation w.r.t. the other component, for which several results on the existence of multiple or infinitely many solutions can be applied. Thus multiple (or infinitely many) trivial solutions to system (1.7) may exist and need to be excluded in numerical computations. Therefore, there is a need to develop some numerical methods for computing those critical points of (1.8) corresponding to the co-existing states to (1.7).

Critical points of a general functional $J$, that are local minima, are the stable solutions and well-studied in calculus of variations. Conventional numerical methods focus on finding such solutions. Critical points that are not local extrema are called saddle points. They appear as unstable equilibria or transient excited states in physical systems, and are traditionally thought to be too hard to capture in practice. 
Highly/multiply excited states, which can now be electronically excited or laser induced, have been observed or demonstrated $[3,11,18,22,27]$ in many fields such as quantum mechanics, condensed matter physics, dynamics of biomolecules, nonlinear optics, etc. Those excited states can easily decay into lower-lying excited states or the ground states with some small perturbations. Meanwhile, those unstable states enjoy a large variety of configurations and maneuverabilities, which may lead to many promising or even surprising applications. With new atomic, optical or synchrotronic technologies, scientists now are able to successfully reach or trap them and search for their potential applications [2,12,24,25,27]. These new technologies and advanced studies are changing the traditional view on unstable solutions and starting to draw more and more attention. As a result, there is a growing need to develop more efficient and reliable numerical methods for finding those multiple excited states, since analytic solution expressions are generally too difficult to obtain.

Spatial solitons have been a subject of many studies $[7,8,13,14,17]$ since their first theoretical prediction [5]. After a number of experimental observations of self-guided light beams in various types of nonlinear bulk media were reported, the study of spatial optical solitons and their interactions became an active research area in nonlinear optics, see $[7,8,11,13,14]$. In particular, it has been shown that several light beams can be combined to produce multicomponent self-trapped states, so-called spatial vector solitons. Physically, these vector solitons (corresponding to co-existing excited states) are "particle-like" localized nonlinear objects; mathematically, they are unstable solitary wave solutions to certain NLS systems $[7,8,11]$, see also (1.1). Since all those vector solitons are unstable, instability analysis becomes important both practically and theoretically. However, "so far, numerical methods have been proved to be the only available tool for analyzing the mutually trapped states in the nonlinear regime, especially solitons without radial symmetry (e.g., dipole or multipole vector solitons)"[8]. It has also been observed that the dipole-mode vector solitons are much more stable than any other vector soliton. They are "stable enough for experimental observation, ..., extremely robust, have a typical lifetime of several hundred diffraction lengths and survive a wide range of perturbations" [11].

Our goal here is to develop some numerical methods for finding those multiple nontrivial solutions (co-existing states) in a stable way and measuring their instabilities as well. So 
far, such methods are not available in the literature. One may want to mention a Newton's method. But it is known that a Newton's method depends so heavily on an initial guess and has difficulties in handling degenerate cases. Besides, even it successfully captures a solution, it is still blind to the instability information of the solution since it does not assume or use the variational structure of a problem. As a pioneering work in variational methods, a mountain pass algorithm [6] was developed to find the ground states (1-saddles) in 1993. Then a high linking method [9] was proposed to capture 2-saddles in 1999. But no mathematical justification on those methods was given. Then, a local minimax method (LMM) together with its mathematical justification and convergence were developed in $[15,16,32]$ to find multiple saddle points in an order based on their instability index [36], i.e., the number of linearly independent directions along which the functional energy decreases under a small perturbation. Meanwhile, LMM views solutions of the form $(0, v)$ or $(u, 0)$ as nontrivial ones on a Cartesian product space. Because of this, our efforts for finding those co-existing states will be greatly weakened or even become fruitless. Therefore new modifications must be developed.

The outline of this paper is as follows. In Section 2 we establish a local min-orthogonal characterization for the co-existing critical points (states) of dual functionals. Based on this characterization, a local min-orthogonal algorithm as well as its convergence for finding multiple co-existing states is given in Section 3. Finally, in Section 4 we implement the algorithm to solve our model problem (1.3) for multiple vector solitons. Due to page limit, results on instability analysis will be presented in a subsequent paper [4].

\section{A New Local Min-Orthogonal Characterization}

In this section, we will establish a general framework for characterizing co-existing saddle points by improving the original framework of LMM. Here is the basic idea of LMM. For a given Hilbert space $H$, one first sets or selects a subspace $L \subset H$, called a support, which is spanned by all trivial and known solutions at lower critical levels and from which an algorithm search needs to keep away; then introduces a composite functional $J(p(u))$ such that $u \in L^{\perp}, p(u) \in H \backslash L$ and $J^{\prime}(p(u)) \perp[L, u]$; and finally seeks a point $u^{*} \in L^{\perp}$ such that $J^{\prime}\left(p\left(u^{*}\right)\right) \perp L^{\perp}$, which implies that $p\left(u^{*}\right)$ is a critical point of $J$ not in $L$. It is easy to see 
that if $p(u)$ is a local maximum of $J$ on $[L, u]$, then $J^{\prime}(p(u)) \perp[L, u]$; on the other hand, if a point $u^{*} \in L^{\perp}$ is a local minimizer of $J(p(u))$ over $L^{\perp}$, then $J^{\prime}\left(p\left(u^{*}\right)\right) \perp L^{\perp}$.

However, when it comes to solving a general cooperative system (1.7) for co-existing solutions, there are usually many and even infinitely many trivial solutions needed to be excluded. Under the above framework, the support $L$ may contain too many trivial solutions in different critical levels and even become infinite dimensional. It causes serious problems in numerical implementation. In this paper, we shall fix this problem by introducing a new selection function and then deriving a new characterization for co-existing saddle points.

For $i=1,2$, let $H_{i}$ be a Hilbert space with inner product $\langle\cdot, \cdot\rangle, L_{i}$ be a closed subspace of $H_{i}$ and $H_{i}=L_{i} \oplus L_{i}^{\perp}$ be the orthogonal decomposition. Denote $H=H_{1} \times H_{2}, L=L_{1} \times L_{2}$. While $L$ is called a "support", $L_{1}, L_{2}$ are called "sub-supports". Then $L_{1}^{\perp} \times L_{2}^{\perp}=L^{\perp}$ and $H=L \oplus L^{\perp}$. Denote $S_{L^{\perp}}=\left\{u \in L^{\perp}:\|u\|=1\right\}$. For $v=\left(v_{1}, v_{2}\right) \in S_{L^{\perp}}$, denote $\left[L_{i}, v_{i}\right]=\left\{t v_{i}+w_{i} \mid w_{i} \in L_{i}, t \in \mathbb{R}\right\}, i=1,2$. Assume $J \in \mathcal{C}^{1}(H, \mathbb{R})$ and denote its gradient by $J^{\prime}=\left(\partial J_{1}, \partial J_{2}\right)$.

Definition 2.1. A set-valued mapping $P: S_{L^{\perp}} \rightarrow 2^{H}$ is called an $L-\perp$ mapping of $J$ if

$$
P(w)=\left\{u \in\left[L_{1}, w_{1}\right] \times\left[L_{2}, w_{2}\right]: \partial J_{i}(u) \perp\left[L_{i}, w_{i}\right], i=1,2\right\} \quad \forall w=\left(w_{1}, w_{2}\right) \in S_{L^{\perp}}
$$

A mapping $p: S_{L^{\perp}} \rightarrow H$ is an $L-\perp$ selection of $J$ if $p(w) \in P(w), \forall w \in S_{L^{\perp}}$. For each $w \in S_{L^{\perp}}$, if $p$ is locally defined near $w$, then $p$ is called a local $L-\perp$ selection of $J$ at $w$.

REMARK 2.1.

(a) Definition 2.1 is stronger than the original one in [35] since

$$
\partial J_{1} \perp\left[L_{1}, w_{1}\right], \partial J_{2} \perp\left[L_{2}, w_{2}\right] \Rightarrow J^{\prime}=\left(\partial J_{1}, \partial J_{2}\right) \perp[L, w], \forall w=\left(w_{1}, w_{2}\right)
$$

However, this new definition not only enables us to identify and capture the co-existing states, but also gives us more flexibility to solve other nonlinear systems.

(b) If $u_{1}$ is a local maximum point of $J\left(\cdot, u_{2}\right)$ in $\left[L_{1}, w_{1}\right]$ and $u_{2}$ is a local maximum point of $J\left(u_{1}, \cdot\right)$ in $\left[L_{2}, w_{2}\right]$, then $p(w)=u=\left(u_{1}, u_{2}\right) \in P(w)$. In such case, $P(p)$ is called a peak mapping (selection) of $J$.

(c) Definition 2.1 can be easily extended to a multicomponent system. 
It is easy to see the orthogonality in the above definition is preserved under a limit of $w$ in $S_{L^{\perp}}$, which leads to the following lemma (its proof is straightforward and thus omitted).

Lemma 2.1. The graph $G=\left\{(u, w): w \in S_{L^{\perp}}, u \in P(w) \neq \emptyset\right\}$ is closed.

With Definition 2.1, critical points of a dual functional $J$ can be characterized as below:

Theorem 2.1. Let $w^{*}=\left(w_{1}^{*}, w_{2}^{*}\right) \in S_{L^{\perp}}$ and $p$ be a local $L-\perp$ selection of $J$ at $w^{*}$ and continuous at $w^{*}$, where $L=L_{1} \times L_{2}$. Assume that $p_{1}\left(w^{*}\right) \notin L_{1}$ and $p_{2}\left(w^{*}\right) \notin L_{2}$, where $p\left(w^{*}\right)=\left(p_{1}\left(w^{*}\right), p_{2}\left(w^{*}\right)\right)$, then a necessary and sufficient condition that $u^{*}=p\left(w^{*}\right)$ is a co-existing critical point of $J$ is that there exists a neighborhood $\mathcal{N}\left(w^{*}\right)$ of $w^{*}$ s.t.

$$
\partial J_{i}\left(p\left(w^{*}\right)\right) \perp p_{i}(w)-p_{i}\left(w^{*}\right), \forall w \in \mathcal{N}\left(w^{*}\right) \cap S_{L^{\perp}}, \quad i=1,2,
$$

where $J^{\prime}\left(p\left(w^{*}\right)\right)=\left(\partial J_{1}\left(p\left(w^{*}\right)\right), \partial J_{2}\left(p\left(w^{*}\right)\right)\right), p(w)=\left(p_{1}(w), p_{2}(w)\right)$.

Proof. Only need to prove the sufficiency. Since $\partial J_{i}\left(p\left(w^{*}\right)\right) \perp L_{i}(i=1,2)$, it suffices to show that $\partial J_{i}\left(p\left(w^{*}\right)\right) \perp L_{i}^{\perp}(i=1,2)$. Let $\mathcal{N}\left(w^{*}\right)$ be a neighborhood of $w^{*}$ s.t. $p$ is well-defined and (2.1) is satisfied. Denote $p_{i}\left(w^{*}\right)=t_{i}^{*} w_{i}^{*}+w_{L_{i}}^{*}$ for some scalar $t_{i}^{*}$ and $w_{L_{i}}^{*} \in L_{i}, i=1,2$. Then $p_{1}\left(w^{*}\right) \notin L_{1}$ and $p_{2}\left(w^{*}\right) \notin L_{2}$ imply $w_{i}^{*} \neq 0$ and $t_{i}^{*} \neq 0, i=1,2$. By the continuity, $p_{i}(w)=t_{i} w_{i}+w_{L_{i}}$ with $t_{i} \neq 0$ and $w_{L_{i}} \in L_{i}(i=1,2)$ for each $w=\left(w_{1}, w_{2}\right) \in \mathcal{N}\left(w^{*}\right) \cap S_{L^{\perp}}$. For $i=1,2$, since $\partial J_{i}\left(p\left(w^{*}\right)\right) \perp\left[L_{i}, w_{i}^{*}\right]$ and $p_{i}\left(w^{*}\right) \in\left[L_{i}, w_{i}^{*}\right]$, we have

$$
\partial J_{i}\left(p\left(w^{*}\right)\right) \perp p_{i}(w)-p_{i}\left(w^{*}\right) \Leftrightarrow \partial J_{i}\left(p\left(w^{*}\right)\right) \perp p_{i}(w) \Leftrightarrow \partial J_{i}\left(p\left(w^{*}\right)\right) \perp w_{i}
$$

for each $w=\left(w_{1}, w_{2}\right) \in \mathcal{N}\left(w^{*}\right) \cap S_{L^{\perp}}$. On the other hand, for each $w \in L^{\perp}$, when the scalar $s$ is small, we have

$$
\frac{w^{*}+s w}{\left\|w^{*}+s w\right\|} \in \mathcal{N}\left(w^{*}\right) \cap S_{L^{\perp}}
$$

This and (2.2) imply that $\partial J_{i}\left(p\left(w^{*}\right)\right) \perp w_{i}(i=1,2), \forall w=\left(w_{1}, w_{2}\right) \in L^{\perp}$, or $\partial J_{i}\left(p\left(w^{*}\right)\right) \perp L_{i}^{\perp}, i=1,2$.

LEMma 2.2. Let $v$ be any unit vector in a normed space $(X,\|\cdot\|)$, then

$$
\left\|v-\frac{v \pm w}{\|v \pm w\|}\right\| \leq \frac{2\|w\|}{\|v \pm w\|}, \forall w \in X \backslash\{v\} .
$$

The following lemma is crucial in this work and will lead to a characterization on coexisting critical points of a dual functional $J$ and a stepsize rule for our numerical algorithm. 
Lemma 2.3. Let $w=\left(w_{1}, w_{2}\right) \in S_{L^{\perp}}$ with $w_{1} \neq 0, w_{2} \neq 0$ and $p$ be a continuous local $L-\perp$ selection of $J$ at $w$. Denote $p(w)=\left(p_{1}(w), p_{2}(w)\right)=\left(t_{1} w_{1}, t_{2} w_{2}\right)+w_{L}$ for some scalars $t_{1}, t_{2}$ and $w_{L} \in L$. If $t_{1} \cdot t_{2}>0$, then either $J^{\prime}(p(w))=(0,0)$ or there exists $s_{0}>0$ s.t.

$$
\begin{aligned}
J(p(w(s)))-J(p(w)) & <-\frac{\min \left\{\left|t_{1}\right|,\left|t_{2}\right|\right\} s\left\|J^{\prime}(p(w))\right\|^{2}}{2 \sqrt{1+s^{2}\left\|J^{\prime}(p(w))\right\|^{2}}} \\
& \leq-\frac{1}{4} \min \left\{\left|t_{1}\right|,\left|t_{2}\right|\right\}\left\|J^{\prime}(p(w))\right\| \cdot\|w(s)-w\|<0,
\end{aligned}
$$

$\forall 0<s<s_{0}$, where $w(s)=\left(w_{1}(s), w_{2}(s)\right)=\frac{w-\operatorname{sign}\left(t_{1}\right) s J^{\prime}(p(w))}{\sqrt{1+s^{2}\left\|J^{\prime}(p(w))\right\|^{2}}} \in S_{L^{\perp}}$ and

$$
p(w(s))=\left(p_{1}(w(s)), p_{2}(w(s))\right)=\left(t_{1}(s) w_{1}(s), t_{2}(s) w_{2}(s)\right)+w_{L}(s)
$$

for some $w_{L}(s) \in L$.

Proof. For convenience, let $d=\left(d_{1}, d_{2}\right)=-J^{\prime}(p(w))$. Since $H=\left(L_{1} \times L_{2}\right) \oplus\left(L_{1}^{\perp} \times L_{2}^{\perp}\right)$ and $w(s) \rightarrow w$ as $s \rightarrow 0, p$ is continuous at $w$ implies that $\|p(w(s))-p(w)\| \rightarrow 0$ and

$$
t_{1}(s) \rightarrow t_{1}, \quad t_{2}(s) \rightarrow t_{2}, \quad \text { as } s \rightarrow 0 .
$$

With $t_{1} \cdot t_{2}>0$ and $J \in \mathcal{C}^{1}(H, \mathbb{R})$, we have

$$
\operatorname{sign}\left(t_{1}(s)\right)=\operatorname{sign}\left(t_{2}(s)\right)=\operatorname{sign}\left(t_{1}\right)=\operatorname{sign}\left(t_{2}\right)
$$

and

$$
\begin{aligned}
& J(p(w(s)))-J(p(w))=\left\langle J^{\prime}(p(w)), p(w(s))-p(w)\right\rangle+o(\|p(w(s))-p(w)\|) \\
= & \left\langle-d_{1}, p_{1}(w(s))-p_{1}(w)\right\rangle+\left\langle-d_{2}, p_{2}(w(s))-p_{2}(w)\right\rangle+o(\|p(w(s))-p(w)\|)
\end{aligned}
$$

when $s$ is small. Next, we note that $p$ is an $L$ - $\perp$ selection, i.e., $d_{1} \perp\left[L_{1}, w_{1}\right], d_{2} \perp\left[L_{2}, w_{2}\right]$. It then follows that $\left\langle d_{1}, p_{1}(w)\right\rangle=\left\langle d_{2}, p_{2}(w)\right\rangle=0$. This together with (2.5) leads to

$$
\begin{aligned}
& \left\langle J^{\prime}(p(w)), p(w(s))-p(w)\right\rangle=\sum_{i=1}^{2}\left\langle-d_{i}, p_{i}(w(s))\right\rangle \\
= & -\sum_{i=1}^{2}\left\langle d_{i}, t_{i}(s) w_{i}(s)\right\rangle=-\sum_{i=1}^{2}\left\langle d_{i}, t_{i}(s) \frac{w_{i}+\operatorname{sign}\left(t_{i}\right) s d_{i}}{\sqrt{1+s^{2}\|d\|^{2}}}\right\rangle\left(\text { since } w_{L}(s) \in L\right) \\
= & -\sum_{i=1}^{2}\left\langle d_{i}, \frac{\operatorname{sign}\left(t_{i}\right) t_{i}(s) s d_{i}}{\sqrt{1+s^{2}\|d\|^{2}}}\right\rangle=-\sum_{i=1}^{2} \frac{\operatorname{sign}\left(t_{i}\right) t_{i}(s) s}{\sqrt{1+s^{2}\|d\|^{2}}}\left\|d_{i}\right\|^{2}\left(\text { since } d_{i} \perp\left[L_{i}, w_{i}\right]\right) \\
= & -\sum_{i=1}^{2} \frac{\operatorname{sign}\left(t_{i}(s)\right) t_{i}(s) s}{\sqrt{1+s^{2}\|d\|^{2}}}\left\|d_{i}\right\|^{2}=-\sum_{i=1}^{2} \frac{\left|t_{i}(s)\right| s\left\|d_{i}\right\|^{2}}{\sqrt{1+s^{2}\|d\|^{2}}} \\
\leq & -\frac{\min \left(\left|t_{1}(s)\right|,\left|t_{2}(s)\right|\right) s}{\sqrt{1+s^{2}\|d\|^{2}}}\|d\|^{2}
\end{aligned}
$$


when $s$ is small. From (2.4), it follows that $\left|t_{1}(s)\right| \rightarrow\left|t_{1}\right|>0,\left|t_{2}(s)\right| \rightarrow\left|t_{2}\right|>0$ and $\min \left\{\left|t_{1}(s)\right|,\left|t_{2}(s)\right|\right\} \rightarrow \min \left\{\left|t_{1}\right|,\left|t_{2}\right|\right\}>0$ as $s \rightarrow 0$. Hence, when $s$ is small, $\min \left\{\left|t_{1}(s)\right|,\left|t_{2}(s)\right|\right\}>\frac{1}{2} \min \left\{\left|t_{1}\right|,\left|t_{2}\right|\right\}>0$. By this and (2.7), there is $s_{0}>0$ s.t.

$$
\left\langle J^{\prime}(p(w)), p(w(s))-p(w)\right\rangle \leq-\frac{\min \left(\left|t_{1}(s)\right|,\left|t_{2}(s)\right|\right) s}{\sqrt{1+s^{2}\|d\|^{2}}}\|d\|^{2}<-\frac{\min \left(\left|t_{1}\right|,\left|t_{2}\right|\right) s}{2 \sqrt{1+s^{2}\|d\|^{2}}}\|d\|^{2}
$$

for $0<s<s_{0}$. On the other hand, by Lemma 2.2 and the orthogonality $d \perp w$, we have

$$
\|w-w(s)\|=\left\|w-\frac{w+\operatorname{sign}\left(t_{1}\right) s d}{\left\|w+\operatorname{sign}\left(t_{1}\right) s d\right\|}\right\| \leq \frac{2 s\|d\|}{\left\|w+\operatorname{sign}\left(t_{1}\right) s d\right\|}=\frac{2 s\|d\|}{\sqrt{1+s^{2}\|d\|^{2}}} .
$$

Combining (2.8) and (2.9), we obtain

$$
\left\langle J^{\prime}(p(w)), p(w(s))-p(w)\right\rangle<-\frac{\min \left(\left|t_{1}\right|,\left|t_{2}\right|\right) s}{2 \sqrt{1+s^{2}\|d\|^{2}}}\|d\|^{2} \leq-\frac{1}{4} \min \left\{\left|t_{1}\right|,\left|t_{2}\right|\right\}\|d\| \cdot\|w(s)-w\| .
$$

This inequality together with (2.6) yields (2.3).

With Lemma 2.3, we can now establish the following local min-orthogonal characterization of the co-existing critical points.

ThEOREM 2.2. If $w=\left(w_{1}, w_{2}\right) \in S_{L^{\perp}}$ and $p(w)=\left(p_{1}(w), p_{2}(w)\right)$ be a local $L-\perp$ selection of $J$ at $w$ s.t. (i) $p$ is continuous at $w$, (ii) $p_{1}(w) \notin L_{1}$ and $p_{2}(w) \notin L_{2}$ and (iii) $w=\arg (l o c) \min _{v \in S_{L^{\perp}}} J(p(v))$, then $p(w)$ is a co-existing critical point of $J$.

Proof. Suppose $J^{\prime}(p(w)) \neq 0$. By (ii), we have $p(w)=\left(p_{1}(w), p_{2}(w)\right)=\left(t_{1} w_{1}, t_{2} w_{2}\right)+w_{L}$ for some scalars $t_{1} \neq 0, t_{2} \neq 0$ and $w_{L} \in L$. There are two cases: either $(\mathrm{a}) t_{1} \cdot t_{2}>0$ or (b) $t_{1} \cdot t_{2}<0$. Since Case (b) can be converted to Case (a) by setting $\tilde{w}=\left(w_{1},-w_{2}\right)$, we only need to discuss Case (a). For Case (a), by Lemma 2.3, there is $s_{0}>0$ s.t. for $0<s<s_{0}$,

$$
J(p(w(s)))<J(p(w))-\frac{1}{4} \min \left\{\left|t_{1}\right|,\left|t_{2}\right|\right\}\left\|J^{\prime}(p(w))\right\| \cdot\|w(s)-w\|<J(p(w)),
$$

which contradicts (iii).

REMARK 2.2. Let us define a solution set $\mathcal{M}=\left\{p(w): w \in S_{L^{\perp}}=S_{L_{1}^{\perp} \times L_{2}^{\perp}}\right\}$. Lemma 2.1 shows that $\mathcal{M}$ is closed and Theorem 2.2 states that a local minimizer of $J(\cdot)$ on $\mathcal{M}$, or $J(p(\cdot))$ on $S_{L^{\perp}}$, yields a saddle point $p\left(w^{*}\right)$, which can be numerically approximated by a minimization method, e.g., a steepest descent method. The subspace $L$ here serves as a support in search of a local minimizer of $J(\cdot)$ outside $L$. 


\section{A Flow Chart of a New Local Min-Orthogonal Algorithm}

In this section, we present a flow chart of our algorithm, called a Local Min-Orthogonal Algorithm (LMOA). With the previous notations, let $\left\{u_{1}, \ldots, u_{m}\right\} \subset H_{1}$ and $\left\{v_{1}, \ldots, v_{n}\right\} \subset$ $H_{2}$ be linearly independent. Denote $L_{1}=\operatorname{span}\left\{u_{1}, \ldots, u_{m}\right\}, L_{2}=\operatorname{span}\left\{v_{1}, \ldots, v_{n}\right\}$ and the support $L=L_{1} \times L_{2}$. Choose an error tolerance $\varepsilon>0$ and a stepsize control parameter $\lambda \in(0,1)$.

Step 1: Set $k=1$. Choose a point $\theta^{(k)}=\left(\theta_{1}^{(k)}, \theta_{2}^{(k)}\right) \in S_{L^{\perp}}$ s.t. $\theta_{1}^{(k)} \neq 0, \theta_{2}^{(k)} \neq 0$ and an appropriate initial guess $\left(t_{0}^{(k)}, \ldots, t_{m}^{(k)}, r_{0}^{(k)}, \ldots, r_{n}^{(k)}\right)$. Use this initial guess to solve a system of $m+n+2$ nonlinear equations

$$
\frac{\partial}{\partial t_{i}^{(k)}} J\left(p\left(\theta^{(k)}\right)\right)=0, \quad \frac{\partial}{\partial r_{j}^{(k)}} J\left(p\left(\theta^{(k)}\right)\right)=0, \text { for } i=0,1, \ldots, m, j=0,1, \ldots, n
$$

for the $m+n+2$ unknowns $\left(t_{0}^{(k)}, \ldots, t_{m}^{(k)}, r_{0}^{(k)}, \ldots, r_{n}^{(k)}\right)$, i.e., to compute an $L$ - $\perp$ selection $p\left(\theta^{(k)}\right)=\left(p_{1}\left(\theta^{(k)}\right), p_{2}\left(\theta^{(k)}\right)\right)$ at $\theta^{(k)}$ s.t.

$$
\begin{aligned}
& p_{1}\left(\theta^{(k)}\right)=\sum_{i=1}^{m} t_{i}^{(k)} u_{i}+t_{0}^{(k)} \theta_{1}^{(k)} \in\left[L_{1}, \theta_{1}^{(k)}\right] \backslash L_{1}, \\
& p_{2}\left(\theta^{(k)}\right)=\sum_{i=1}^{n} r_{i}^{(k)} v_{i}+r_{0}^{(k)} \theta_{2}^{(k)} \in\left[L_{2}, \theta_{2}^{(k)}\right] \backslash L_{2},
\end{aligned} \quad \text { and } t_{0}^{(k)} r_{0}^{(k)}>0 .
$$

Step 2: Set $w^{(k)}=p\left(\theta^{(k)}\right)$ and compute the gradient $d^{(k)}=\left(d_{1}^{(k)}, d_{2}^{(k)}\right)=J^{\prime}\left(w^{(k)}\right)$.

Step 3: If $\left\|d^{(k)}\right\|<\varepsilon$, then OUTPUT $w^{(k)}$, STOP; Otherwise, GOTO Step 4.

Step 4: For each $s>0$, let $\theta^{(k)}(s)=\left(\theta_{1}^{(k)}(s), \theta_{2}^{(k)}(s)\right)=\frac{\theta^{(k)}-\operatorname{sign}\left(t_{0}^{(k)}\right) s d^{(k)}}{\left\|\theta^{(k)}-\operatorname{sign}\left(t_{0}^{(k)}\right) s d^{(k)}\right\|}$.

Determine the stepsize

$$
\begin{aligned}
s^{k}= & \max _{i \in \mathbb{N}}\left\{\frac{\lambda}{2^{i}} \mid 2^{i}>\left\|d^{(k)}\right\|,\right. \\
& \left.J\left(p\left(\theta^{(k)}\left(\frac{\lambda}{2^{i}}\right)\right)\right)-J\left(w^{(k)}\right)<-\frac{1}{4} \min \left\{\left|t_{0}^{(k)}\right|,\left|r_{0}^{(k)}\right|\right\}\left\|d^{(k)}\right\| \cdot\left\|\theta^{(k)}\left(\frac{\lambda}{2^{i}}\right)-\theta^{(k)}\right\|\right\},
\end{aligned}
$$

where $\left(t_{0}^{(k)}, t_{1}^{(k)}, \ldots, t_{m}^{(k)}, r_{0}^{(k)}, r_{1}^{(k)}, \ldots, r_{n}^{(k)}\right)$ is used as an initial guess to evaluate $p\left(\theta^{(k)}\left(\frac{\lambda}{2^{i}}\right)\right)=\left(p_{1}\left(\theta^{(k)}\left(\frac{\lambda}{2^{i}}\right)\right), p_{2}\left(\theta^{(k)}\left(\frac{\lambda}{2^{i}}\right)\right)\right)$ with $p_{1}\left(\theta^{(k)}\left(\frac{\lambda}{2^{i}}\right)\right) \notin L_{1}$ and $p_{2}\left(\theta^{(k)}\left(\frac{\lambda}{2^{i}}\right)\right) \notin L_{2}$ in the same way as in Step 1.

Step 5: Set $\theta^{(k+1)}=\theta^{(k)}\left(s^{k}\right), p\left(\theta^{(k+1)}\right)=p\left(\theta^{(k)}\left(s^{k}\right)\right)$ and $k \leftarrow k+1$. GOTO Step 2 . 


\section{REMARK 3.1.}

(a) The algorithm generally starts from the case $L_{1}=L_{2}=\{0\}$. With different initial points in $S_{L^{\perp}}$, it can find one or more saddle points (solutions), say $\left(u_{1}^{1}, v_{1}^{1}\right)$, $\left(u_{1}^{2}, v_{1}^{2}\right), \ldots,\left(u_{1}^{e_{1}}, v_{1}^{e_{1}}\right)$. Then gradually increasing $L_{1}$ and $L_{2}$, respectively, (for example, with a fixed $L_{1}$ contained in $\operatorname{span}\left\{u_{1}^{1}, u_{1}^{2}, \ldots, u_{1}^{e_{1}}\right\}$, one can gradually increase $L_{2}$ by adding more and more linearly independent elements from $\left.\operatorname{span}\left\{v_{1}^{1}, v_{1}^{2}, \ldots, v_{1}^{e_{1}}\right\}\right)$, it then captures one or more higher order saddle points $\left(u_{2}^{1}, v_{2}^{1}\right),\left(u_{2}^{2}, v_{2}^{2}\right), \ldots,\left(u_{2}^{e_{2}}, v_{2}^{e_{2}}\right)$. By this way, multiple branches of solutions can be located. On the other hand, if a problem possesses certain symmetries, one may easily construct the sub-supports $L_{1}$ and $L_{2}$ by applying those symmetries, refer to Section 4.2 .

(b) The $m+n+2$ equations in (3.1) come exactly from the definition of an $L$ - $\perp$ selection, i.e., $\partial J_{1}\left(p\left(\theta^{(k)}\right)\right) \perp\left[L_{1}, \theta_{1}^{(k)}\right], \partial J_{2}\left(p\left(\theta^{(k)}\right)\right) \perp\left[L_{2}, \theta_{2}^{(k)}\right]$ and are apparently satisfied for any critical point of $J$. To find a new critical point, one needs to choose an appropriate initial guess in Step 1 (for example, except $t_{0}^{(1)}, r_{0}^{(1)}$, set all other entries $\left.t_{1}^{(1)}=\ldots=t_{m}^{(1)}=r_{1}^{(1)}=\ldots=r_{n}^{(1)}=0\right)$, then use this initial guess to find a solution, still denoted by $\left(t_{0}^{(1)}, t_{1}^{(1)}, \ldots, t_{m}^{(1)}, r_{0}^{(1)}, r_{1}^{(1)}, \ldots, r_{n}^{(1)}\right)$, of $(3.1)$ s.t. $t_{0}^{(1)} \neq 0, r_{0}^{(1)} \neq 0$, i.e., $p_{1}\left(\theta^{(1)}\right) \notin L_{1}, p_{2}\left(\theta^{(1)}\right) \notin L_{2}$. Then this solution will be used as the initial guess for next evaluation of $p$. In Step 4, it is crucial to follow the initial guess $\left(t_{0}^{(k)}, t_{1}^{(k)}, \ldots, t_{m}^{(k)}, r_{0}^{(k)}, r_{1}^{(k)}, \ldots, r_{n}^{(k)}\right)$ consistently in evaluating the $L-\perp$ selection $p$ from equations in (3.1). Such a strategy is used to avoid a possible jump of $p$ from one branch to another in the solution set $\mathcal{M}$ and to keep $p$ "continuous". Note that equations in (3.1) usually have multiple solutions when $J$ has multiple critical points.

(c) The algorithm is stable in the sense that the energy functional $J$ is strictly decreasing, i.e., $J\left(p\left(\theta^{(k+1)}\right)\right)<J\left(p\left(\theta^{(k)}\right)\right)$.

To obtain a convergence result of LMOA, the Palais-Smale (PS) condition is needed in replace of the usual compactness condition.

Definition 3.1. A functional $J \in \mathcal{C}^{1}(X, \mathbb{R})$ is said to satisfy the Palais-Smale (PS) condition if any sequence $\left\{u_{i}\right\} \subseteq X$ s.t. $J\left(u_{i}\right)$ is bounded and $J^{\prime}\left(u_{i}\right) \rightarrow 0$ possesses a convergent subsequence. 
Then a subsequence convergence result parallel to Theorems 3.1-3.2 in [16] reads as:

Theorem 3.1. Let $L_{i}$ be a closed subspace of $H_{i}, i=1,2, L=L_{1} \times L_{2}$. Assume $J \in \mathcal{C}^{1}\left(H_{1} \times H_{2}, \mathbb{R}\right)$ satisfies the PS condition and $p$ is an $L-\perp$ selection of $J$ s.t. (i) $p$ is continuous, (ii) $\operatorname{dist}\left(p_{i}\left(\theta^{(k)}\right), L_{i}\right)>\alpha>0(i=1,2)$ for some $\alpha>0$ and all $k=1,2, \ldots$, and (iii) $\inf _{1 \leq k<\infty} J\left(p\left(\theta^{(k)}\right)\right)>-\infty$, where $\left\{p\left(\theta^{(k)}\right) \equiv\left(p_{1}\left(\theta^{(k)}\right), p_{2}\left(\theta^{(k)}\right)\right)\right\}$ is a sequence generated by LMOA (wherein the stop criterion $\left\|d^{(k)}\right\|<\varepsilon$ is replaced by $\left.\left\|d^{(k)}\right\|=0\right)$. If denote $w^{(k)}=p\left(\theta^{(k)}\right)$, then

(a) $\left\{w^{(k)}\right\}$ possesses a subsequence converging to a co-existing critical point of $J$,

(b) any convergent subsequence of $\left\{w^{(k)}\right\}$ converges to a co-existing critical point of J.

Proof. Follows the same lines as in the proof of Theorems 3.1-3.2 in [16] for a Cartesian product space and applies condition (ii).

Similarly, more convergence results can be established as in [16]. Since LMOA is based on a steepest descent method, its rate of convergence is expected to be linear. To speed up the convergence, a Newton's method can be used after a number of iterations by LMOA, refer also to [28].

\section{Application for Solving Multiple Spatial Vector Solitons}

With the algorithm proposed in Section 3, we are ready to carry out some numerical computations for our model problem (1.3). Let $H_{1}=H_{2}=H_{0}^{1}(\Omega)$ and $H=H_{1} \times H_{2}$. We denote the coordinates $(x, y)$ by $x=\left(x_{1}, x_{2}\right)$. By previous considerations, we have

$G_{u}(x, u(x), v(x))=G_{u}(u(x), v(x))=-u(x)+\frac{u(x) I(x)}{1+\mu I(x)}=\frac{1-\mu}{\mu} u(x)-\frac{u(x)}{\mu(1+\mu I(x))}$,
$G_{v}(x, u(x), v(x))=G_{v}(u(x), v(x))=-\gamma v(x)+\frac{v(x) I(x)}{1+\mu I(x)}=\frac{1-\gamma \mu}{\mu} v(x)-\frac{v(x)}{\mu(1+\mu I(x))}$, and

$$
G(x, u(x), v(x))=G(u(x), v(x))=\frac{1-\mu}{2 \mu} u^{2}(x)+\frac{1-\gamma \mu}{2 \mu} v^{2}(x)-\frac{\ln (1+\mu I(x))}{2 \mu^{2}}
$$

Thus system (1.3) is of the form (1.7) and its dual functional is of the form (1.8). While co-existing solutions to system (1.3) have been repeatedly observed in experiments [11, 13], most existence results (see, e.g., [1, 21, 33, 37]) in mathematics literature only focus on 
nonzero solutions not the co-existing ones. Furthermore, a closer examination of system (1.3) displays the following facts.

Proposition 4.1. (a) Any solution $(u, v)$ to (1.3) with $\gamma \in(0,1)$ satisfies $u \perp v$ in $L^{2}(\Omega)$ or $\int_{\Omega} u(x) v(x) d x=0$. (b) If $\gamma \in(0,1]$, then (1.3) has a positive co-existing solution $(u, v)$ only if $\gamma=1$;

Proof. Multiplying the first and the second equations in (1.3) respectively by $v$ and $u$, and integrating by parts yields

$$
\left\{\begin{array}{l}
\int_{\Omega}\left[\Delta u(x) \cdot v(x)-u(x) v(x)+\frac{u(x) v(x) I(x)}{1+\mu I(x)}\right] d x=0 \\
\int_{\Omega}\left[\Delta u(x) \cdot v(x)-\gamma v(x) u(x)+\frac{v(x) u(x) I(x)}{1+\mu I(x)}\right] d x=0 .
\end{array}\right.
$$

After subtraction, one obtains $(\gamma-1) \int_{\Omega} u(x) v(x) d x=0$. Then, (a) follows if $\gamma \in(0,1)$ and (b) follows if $\gamma \in(0,1]$ and $u>0, v>0$.

The orthogonality condition in Proposition 4.1(a) gives us a hint on selecting initial guesses for our algorithm, i.e., we choose initial guesses $(u, v)$ such that $u \perp v$ in $L^{2}(\Omega)$, refer to those initial guesses provided in Section 4.2 (except the initial guesses for cases (3) and (4)). In addition, Proposition 4.1(b) coincides with the experimental observations, "...because the state $|0,0\rangle$, nodeless in both components, can exist only in the degenerate case $\gamma=1$, when ..."[20].

\subsection{Computation of the Gradient $\nabla J(w)$ and an $L-\perp$ Selection $p(\theta)$}

In this subsection, we discuss how to compute $\nabla J(w)$ and an $L-\perp$ selection $p(\theta)$ for (1.7) and (1.8). Let $\|\cdot\|$ be the norm of $H_{1}=H_{2}=H_{0}^{1}(\Omega)$ defined by the inner product $\langle u, v\rangle=\int_{\Omega} \nabla u(x) \cdot \nabla v(x) d x, \forall u, v \in H_{0}^{1}(\Omega)$. Then for $H=H_{1} \times H_{2}$, its inner product is $\left\langle w_{1}, w_{2}\right\rangle=\left\langle u_{1}, u_{2}\right\rangle+\left\langle v_{1}, v_{2}\right\rangle$ and norm is $\left\|w_{1}\right\|^{2}=\left\|u_{1}\right\|^{2}+\left\|v_{1}\right\|^{2}$, where $w_{1}=\left(u_{1}, v_{1}\right), w_{2}=\left(u_{2}, v_{2}\right) \in H$. For $J$ as in (1.8), its Frechet derivative at $\bar{w}=(\bar{u}, \bar{v})$ is

$$
J^{\prime}(\bar{w})=\left(-\Delta \bar{u}-G_{u}(x, \bar{u}, \bar{v}),-\Delta \bar{v}-G_{v}(x, \bar{u}, \bar{v})\right) \in W^{-1,2}(\Omega) \times W^{-1,2}(\Omega),
$$

whose smoothness is "poor". In general, it cannot be used as a search direction in $H_{0}^{1}(\Omega)=W^{1,2}(\Omega)$. Thus we use its canonical identification in $H$ to define the gradient

$$
\nabla J(\bar{w})=\Delta^{-1}\left(-J^{\prime}(\bar{w})\right)=\left(\bar{u}+\Delta^{-1}\left(G_{u}(x, \bar{u}, \bar{v})\right), \bar{v}+\Delta^{-1}\left(G_{v}(x, \bar{u}, \bar{v})\right)\right) \in H,
$$


which implies

$$
J^{\prime}(\bar{w})=(0,0) \Leftrightarrow \nabla J(\bar{w})=(0,0) \Leftrightarrow \bar{w} \text { is a critical point of } J .
$$

Denote $d=\left(d_{1}, d_{2}\right)=\nabla J(\bar{w})$. Then $d$ can be solved from the following linear elliptic system

$$
\left\{\begin{aligned}
\Delta d_{1}(x) & =\Delta \bar{u}(x)+G_{u}(x, \bar{u}(x), \bar{v}(x)), & & x \in \Omega \\
\Delta d_{2}(x) & =\Delta \bar{v}(x)+G_{v}(x, \bar{u}(x), \bar{v}(x)), & & x \in \Omega \\
d_{1}(x) & =d_{2}(x)=0, & & x \in \partial \Omega,
\end{aligned}\right.
$$

e.g., by a MATLAB subroutine ASSEMPDE, a linear PDE or PDE systems solver based on a finite-element method.

In Steps 1 and 4 of LMOA, we need to compute $w=p(\theta)$, the value of a local $L$ - $\perp$ selection $p$ at a point $\theta=\left(\theta_{1}, \theta_{2}\right) \in S_{L_{1}^{\perp} \times L_{2}^{\perp}}$, where $L_{1}=\operatorname{span}\left\{u_{1}, \ldots, u_{m}\right\}$ and $L_{2}=\operatorname{span}\left\{v_{1}, \ldots, v_{n}\right\}$. From the definition of $p$, we can write $w=\left(w_{1}, w_{2}\right)=\left(t_{0} \theta_{1}+\sum_{i=1}^{m} t_{i} u_{i}, r_{0} \theta_{2}+\sum_{i=1}^{n} r_{i} v_{i}\right)$. Here, the $m+n+2$ unknowns $t_{0}, t_{1}, \ldots, t_{m}, r_{0}, r_{1}, \ldots, r_{n}$ are solved from the orthogonal conditions $\partial J_{1}(w) \perp\left[L_{1}, \theta_{1}\right], \partial J_{2}(w) \perp\left[L_{2}, \theta_{2}\right]$, which, through integration by parts, lead to a system of $m+n+2$ nonlinear algebraic equations

$$
\left\{\begin{array}{l}
\int_{\Omega}\left[\Delta w_{1}(x)+G_{u}\left(x, w_{1}(x), w_{2}(x)\right)\right] \theta_{1} d x=0, \\
\int_{\Omega}\left[\Delta w_{1}(x)+G_{u}\left(x, w_{1}(x), w_{2}(x)\right)\right] u_{i} d x=0, \quad i=1, \ldots, m, \\
\int_{\Omega}\left[\Delta w_{2}(x)+G_{v}\left(x, w_{1}(x), w_{2}(x)\right)\right] \theta_{2} d x=0, \\
\int_{\Omega}\left[\Delta w_{2}(x)+G_{v}\left(x, w_{1}(x), w_{2}(x)\right)\right] v_{j} d x=0, \quad j=1, \ldots, n .
\end{array}\right.
$$

This system is then solved by a MATLAB subroutine FSOLVE or FMINUNC with an initial guess selected by the same strategy as described in Remark 3.1(b).

\subsection{Numerical Results}

Due to the localized nature $[8,11,20]$ of stationary states to system (1.1), i.e., $u(x), v(x)$ vanish as $\|x\| \rightarrow \infty$, we set $\Omega=(-10,10) \times(-10,10)$ as in [11, 31]. Also, we choose $\gamma=0.65, \mu=0.5$ as in [11] for system (1.3) and set the error tolerance $\varepsilon=10^{-4}$ to terminate our iteration. Since our model problem (1.1) possesses various symmetries, we developed a symmetric mesh grid on $\Omega$. Fig. 1(a) is a coarse sample of a symmetric mesh we used. 


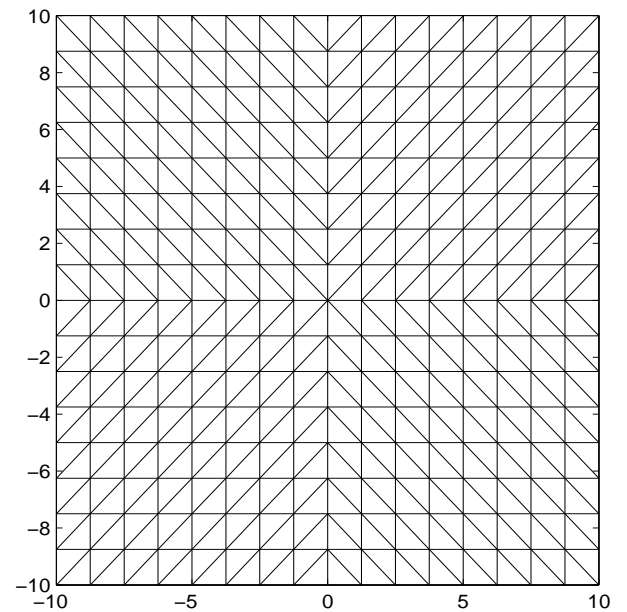

(a)
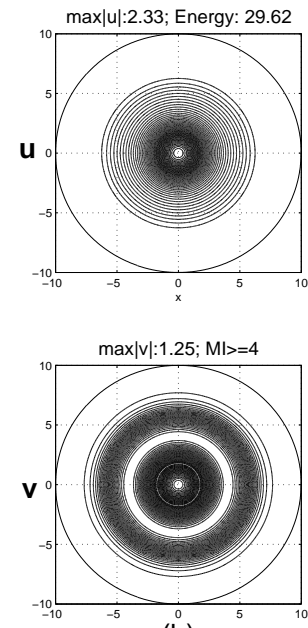

(b)

FIG. 1. (a) A sample symmetric mesh on the square domain $\Omega=(-10,10) \times(-10,10)$; (b) $A$ vortex mode vector soliton/solution to (1.3) on a radial domain.

As an important notion in stability analysis, the Morse index (MI) [26] reveals some information on the local structures of nondegenerate critical points and has been widely applied to measure local instabilities of unstable solutions (saddle points). As a generalization, a local instability index (LII) $\left(\operatorname{LII}\left(u^{*}, v^{*}\right)=\operatorname{dim}\left(L_{1}\right)+\operatorname{dim}\left(L_{2}\right)+\right.$ $\left.\operatorname{dim}\left(\operatorname{span}\left\{u^{*}\right\}\right)+\operatorname{dim}\left(\operatorname{span}\left\{v^{*}\right\}\right)\right)$ associated with our algorithm is defined in [4] to induce a partial order of multiple solutions captured. Fig. 2 lists the first few co-existing states (corresponding to dipole- and multipole-mode vector solitons) to system (1.3) in such a partial order. Solutions (a) and (b), (c) and (d), (e) and (f), etc., have the same LII, respectively. Note that solutions (a) and (b) correspond to the so-called dipole-mode vector solitons with $45^{\circ}$ orientation and horizontal orientation, respectively; while solutions (e)(f) correspond to two quadru-mode vector solitons with different orientations. Physically, dipole-mode vector solitons are the most stable vector solitons. According to [4], they have the least MI $(\geq 3)$ among all the co-existing states. Hence, mathematically, dipole-mode solitons are still unstable, though "they are extremely robust objects, ..., survive a wide range of perturbations" [11]. Besides, it can be seen from Fig. 2 that for each solution $(u, v)$, the 2nd component (i.e., $v$-component) always carries more complex solution structures than the 1st component (i.e., $u$-component) does. To be more precise, one can see that the $v$ component always has at least the same number of nodal lines as the $u$-component does. 
Take solution (i) or (j), for example, when $u$-component is nodal, then $v$-component must be nodal as well; conversely, $v$-component cannot be nodeless (i.e., bell-shaped) when $u$ component is nodal. In physics, $u$-component is called a fundamental mode (wave). It traps and guides a weaker higher-order mode ( $v$-component) while traveling in a nonlinear bulk medium [7].

The orthogonality condition stated in Proposition 4.1(a) enables us to construct the following supports $L$ 's and initial guesses $\left(u_{0}(x, y), v_{0}(x, y)\right)$ 's to compute multiple co-existing states of system (1.3). For a desired solution of large Morse index with certain symmetries, it is more advantageous and efficient to apply such symmetries as carrying out in [29]. More precisely, we use the Haar projection to preserve the symmetries, keeping or forcing our iterations in an invariant subspace induced by such symmetries. Even so a nonzero support sometimes is still required. Take the triple-hump vector solitons (refer to Fig. 2(g) or (h)), for example, we need a bell-shaped symmetric function (symmetric w.r.t. both the $x$ and $y$ axes) which serves as a sub-support for the $v$-component no matter what kind of symmetry we impose on that component. This is because for a triple-hump nodal function as in the second component of solution (g) or (h), without a sub-support, eventually it will collapse, i.e., it will decay to zero. Thus, as long as multiple solutions are concerned, introducing a support is crucial in numerical computations. Contrary to a Newton's method, the selection of an initial guess in our method is quite flexible. However, for the instructive purpose and readers' convenience, suggested initial guesses $\left(u_{0}, v_{0}\right)$ 's and supports $L$ 's are included below, wherein $f(x, y)=e^{-0.05\left(x^{2}+y^{2}\right)}\left(y^{2}-100\right)\left(x^{2}-100\right), g(x, y)=\cos (0.05 \pi x) \cos (0.05 \pi y)$. It is easy to check that $f(x, y), g(x, y)$ are positive symmetric functions on $\Omega$.

(1) cf. Fig.2(a). Choose $\left(u_{0}, v_{0}\right)=(f(x, y), f(x, y)(y+x))$ and $L=\{0\} \times\left\{w_{1}\right\}$ with $w_{1}=e^{-\frac{x^{2}+y^{2}}{50}} g(x, y)$. Solution (a) can also be obtained by applying the odd symmetry to the $v$-component w.r.t. the line $y+x=0$ while letting $L=\{0\} \times\{0\}$. This is the most stable co-existing state that we can find. Its Morse index is at least 3.

(2) cf. Fig.2(b). Choose $\left(u_{0}, v_{0}\right)=(f(x, y), f(x, y) x) . L$ is the same as in case (1). Solution (b) can also be obtained by applying the odd symmetry to the $v$-component w.r.t. the $y$-axis while letting $L=\{0\} \times\{0\}$. Its Morse index is at least 3 . 
Computational Theory and Method for Nonlinear Systems
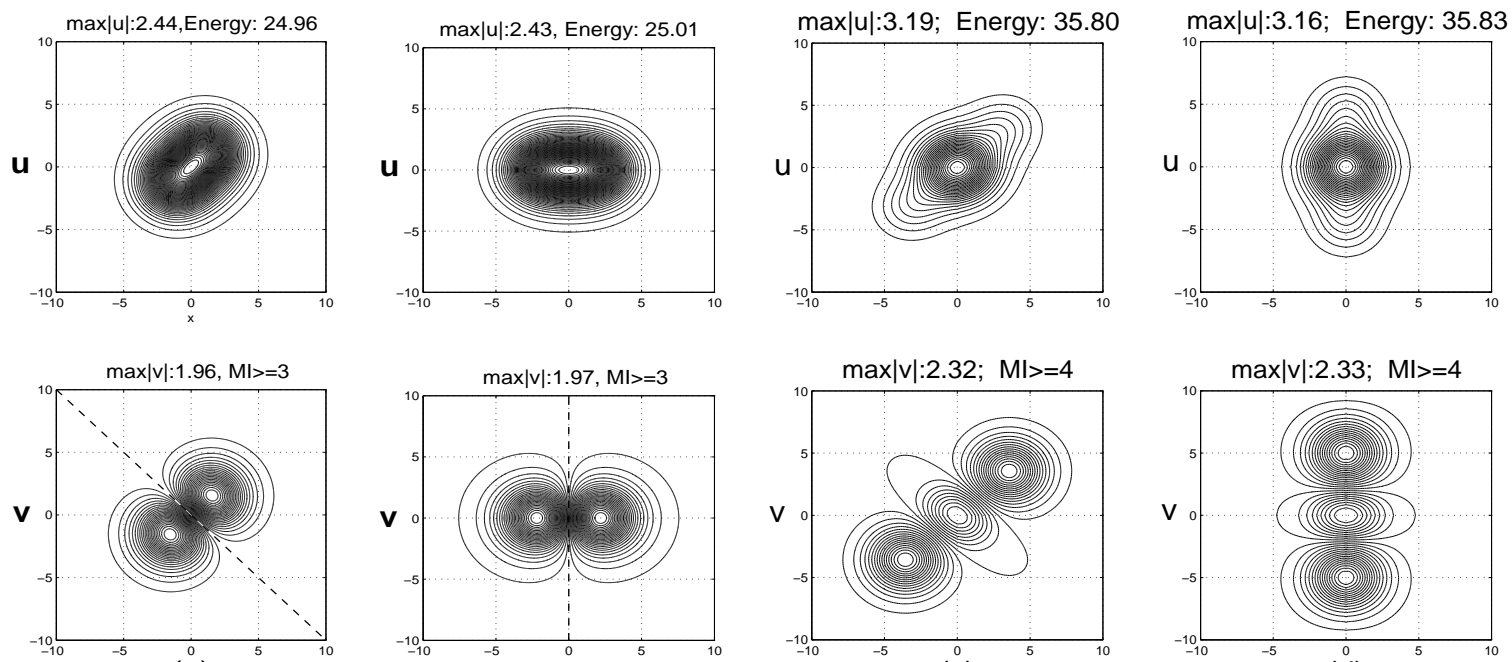

(b)

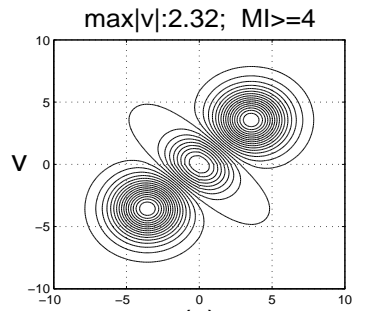

(c)

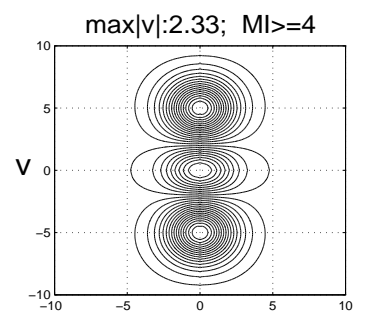

(d)
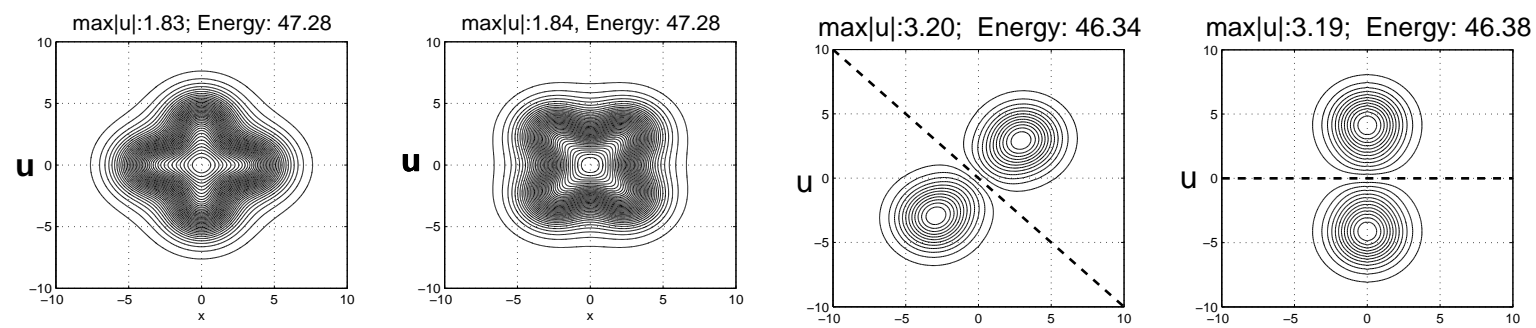

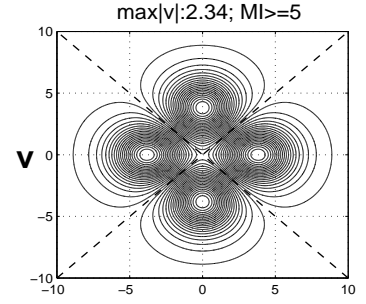

(e)
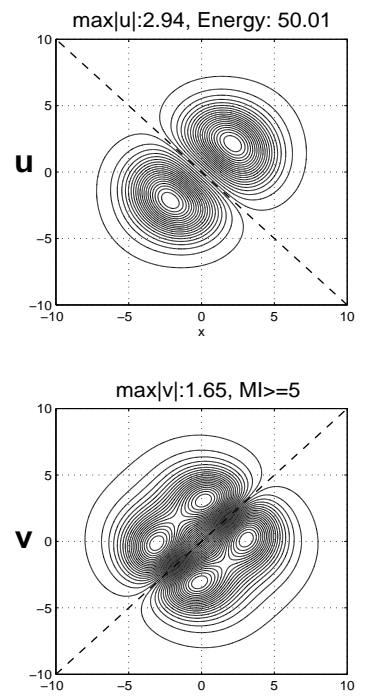

(i)

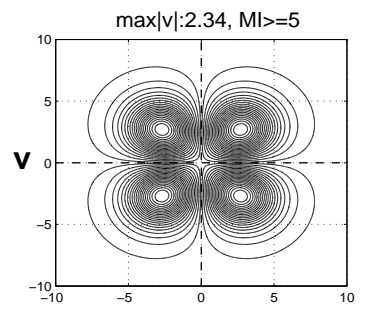

(f)
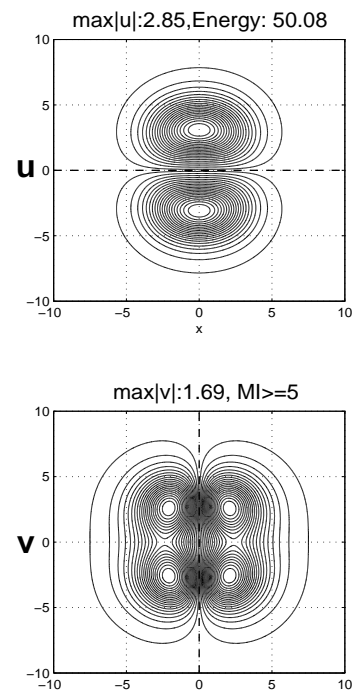

(j)

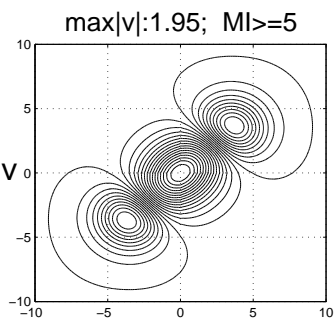

(g)
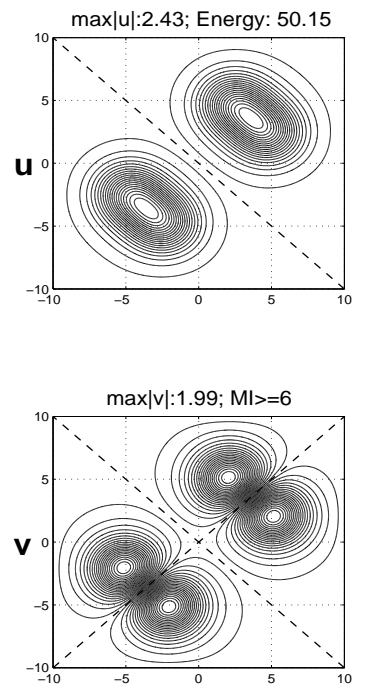

(k)

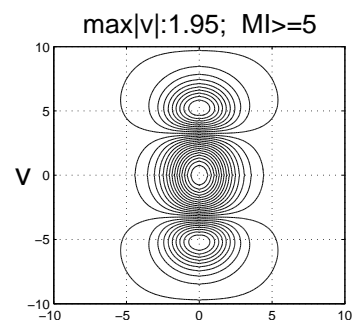

(h)
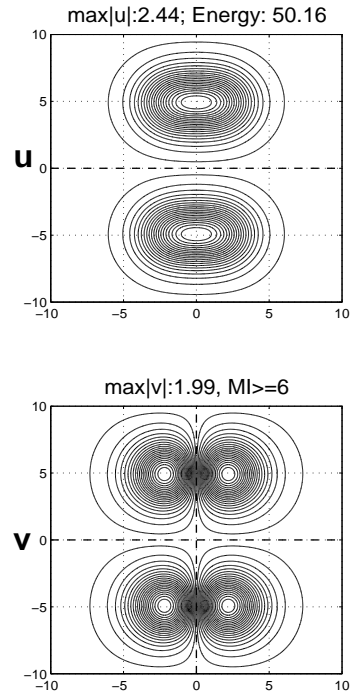

(I)

FIG. 2. Dipole- and multipole-mode vector solitons/solutions to (1.3) on a square domain. Dash lines indicate the nodal lines. 
(3) cf. Fig.2(c). Choose $\left(u_{0}, v_{0}\right)=g(x, y)\left(e^{\frac{(x+y)^{2}}{50}-\frac{x^{2}+y^{2}}{20}}, e^{-\frac{(x+y)^{2}}{45}}\left(y+x+\frac{10}{3}\right)\left(y+x-\frac{10}{3}\right)\right)$ and $L=\{0\} \times\left\{w_{1}, w_{2}\right\}$ with $w_{1}=e^{-\frac{(x+y)^{2}}{45}} g(x, y), w_{2}=(y+x) w_{1}$. This solution can also be found by applying the even symmetry w.r.t. the line $y+x=0$ to the $v$-component while letting $L=\{0\} \times\left\{w_{1}\right\}$. Here, the sub-support $\left\{w_{1}\right\}$ for the $v$-component is essential; without it, the $v$-component will collapse (i.e., decay to zero), which one needs to avoid in order to obtain the co-existing solution. Note that the $v$-component is a tripe-hump sign changing function. The MI of solution (c) is at least 4.

(4) cf. Fig.2(d). Choose $u_{0}=e^{\frac{y^{2}}{25}-\frac{x^{2}+y^{2}}{20}} g(x, y), v_{0}=e^{-\frac{(x+y)^{2}}{45}} g(x, y)\left(y+\frac{10}{3}\right)\left(y-\frac{10}{3}\right)$ and $L=\{0\} \times\left\{w_{1}, w_{2}\right\}$ with $w_{1}=e^{-\frac{(x+y)^{2}}{45}} g(x, y), w_{2}=e^{-\frac{(x+y)^{2}}{45}} \cos (0.05 \pi x) \sin (0.1 \pi y)$. Similarly, solution (d) can be found by applying the even symmetry w.r.t. the $x$ axis to the $v$-component while letting $L=\{0\} \times\left\{w_{1}\right\}$. Again, the $v$-component is a tripe-hump sign changing function for which the sub-support $\left\{w_{1}\right\}$ is necessary. The associated Morse index is at least 4.

(5) cf. Fig.2(e). Choose $\left(u_{0}, v_{0}\right)=f(x, y)\left(1, y^{2}-x^{2}\right)$ and $L=\{0\} \times\left\{w_{1}, w_{2}, w_{3}\right\}$ with

$$
w_{1}=e^{-\frac{x^{2}+y^{2}}{50}} g(x, y), w_{2}=e^{-\frac{x^{2}+y^{2}}{40}} g(x, y)(y-x), w_{3}=e^{-\frac{x^{2}+y^{2}}{40}} g(x, y)(y+x) .
$$

Likewise, solution (e) can be obtained by letting $L=\{0\} \times\{0\}$ and applying the odd symmetry to the $v$-component w.r.t. both the line $y-x=0$ and the line $y+x=0$. This is a quadrupole mode vector soliton whose Morse index is at least 5.

(6) cf. Fig.2(f). Choose $\left(u_{0}, v_{0}\right)=\left(e^{-\frac{x^{2}+y^{2}}{25}}\left(y^{2}-100\right)\left(x^{2}-100\right), f(x, y) x y\right)$ and $L=\{0\} \times$ $\left\{w_{1}, w_{2}, w_{3}\right\}$ with $w_{1}=e^{-\frac{x^{2}+y^{2}}{50}} g(x, y), w_{2}=e^{-\frac{x^{2}+y^{2}}{40}} g(x, y) x, w_{3}=e^{-\frac{x^{2}+y^{2}}{40}} g(x, y) y$. Likewise, solution (f) can be obtained by letting $L=\{0\} \times\{0\}$ and applying the odd symmetry to the $v$-component w.r.t. both the $x$-axis and the $y$-axis. This is another quadrupole mode vector soliton whose Morse index is at least 5 .

(7) cf. Fig.2(g). Choose $\left(u_{0}, v_{0}\right)=\left(e^{-\frac{x^{2}+y^{2}}{10}}\left(y^{2}-100\right)\left(x^{2}-100\right)(y+x), f(x, y) x y\right)$ and apply the even (odd) symmetry w.r.t. the line $y+x=0$ to the $v$ - $(u-)$ component, respectively. Meanwhile, a support $L=\{0\} \times\left\{w_{1}\right\}$ with $w_{1}=e^{-\frac{x^{2}+y^{2}}{40}} g(x, y)$ is used. Again, the $v$-component is a tripe-hump sign changing function for which the subsupport $\left\{w_{1}\right\}$ is required. The associated Morse index is at least 5 . 
(8) cf. Fig.2(h). Choose $u_{0}=e^{-\frac{x^{2}+y^{2}}{15}} \sin \left(\frac{\pi y}{10}\right) \cos \left(\frac{\pi x}{20}\right), v_{0}=e^{-\frac{x^{2}+y^{2}}{20}} g(x, y)\left(y^{2}-x^{2}\right)$ and apply the even (odd) symmetry w.r.t. the $x$-axis to the $v$ - $(u$-) component, respectively. Meanwhile, a support $L=\{0\} \times\left\{w_{1}\right\}$ with $w_{1}=e^{-\frac{x^{2}+y^{2}}{50}} g(x, y)$ is used. Likewise, the $v$-component is a tripe-hump sign changing function for which the sub-support $\left\{w_{1}\right\}$ is essential. The associated Morse index is at least 5 .

(9) cf. Fig.2(i). Choose $\left(u_{0}, v_{0}\right)=(f(x, y)(y+x), f(x, y)(y-x)), L=\{0\} \times\{0\}$ and apply the odd symmetry w.r.t. the line $y+x=0$ to the $u$-component and the odd (even) symmetry w.r.t. the line $y-x=0(y+x=0)$ to the $v$-component. This solution corresponds to a dipole-dipole mode vector soliton with Morse index at least 5.

(10) cf. Fig.2(j). Choose $\left(u_{0}, v_{0}\right)=(f(x, y) y, f(x, y) x), L=\{0\} \times\{0\}$ and apply the odd symmetry w.r.t. the $x$-axis to the $u$-component and the odd (even) symmetry w.r.t. the $y$-axis ( $x$-axis) to the $v$-component. This is another dipole-dipole mode vector soliton with Morse index at least 5.

(11) cf. Fig.2(k). Choose $\left(u_{0}, v_{0}\right)=f(x, y)(y+x)(1, y-x)$ and $L=\{0\} \times\{0\}$. Then, apply the odd symmetry w.r.t. the line $y+x=0$ to the $u$-component, and the odd symmetry w.r.t. both the lines $y+x=0$ and $y-x=0$ to the $v$-component, respectively. Its Morse index is at least 6.

(12) cf. Fig.2(l). Choose $\left(u_{0}, v_{0}\right)=f(x, y) y(1, x)$ and $L=\{0\} \times\{0\}$. Then, apply the odd symmetry w.r.t. the $x$-axis to the $u$-component, and the odd symmetry w.r.t. both the $x$ and $y$ axes to the $v$-component, respectively. Its Morse index is at least 6 .

To obtain a vortex-mode vector soliton, we use a radial domain $\tilde{\Omega}=\left\{(x, y): x^{2}+y^{2}<\right.$ $100\}$. A vortex-mode vector soliton on $\tilde{\Omega}$ is shown as in Fig. 1(b), where the $v$-component is a sign changing radial function with a 1-dimensional peak set (ring-shaped). For such a vortex structure, there is no finite dimensional sufficient support available due to the radial symmetry. A local min-orthogonal approximation, followed by a Newton's iteration method, can capture such a radial solution. Hence, it should possess a large MI (at least 4) and be more unstable than the dipole-mode vector soliton which has the least LII (and MI) among all the vector solitons. This analysis coincides with the observation made in [11], i.e., "..., 
with a small perturbation, the system of a vortex structure (vortex soliton) near cutoff will decay into the system of a dipole structure (dipole soliton)".

Except those co-existing solutions to (1.3), there are quite a few non co-existing ones, which are of less interest in physics applications and hence are omitted here. With our new local min-orthogonal method, we are able to locate the co-existing solutions while excluding the non co-existing ones. More investigation on computational theory and methods for solving nonlinear elliptic systems will be presented in a subsequent paper [4].

\section{Acknowledgement}

The second author's research is supported in part by NSF DMS-0311905. The authors thank two anonymous reviewers for their helpful comments.

\section{References}

[1] T. Bartsch, K.-C. Chang and Z.-Q. Wang, On the Morse indices of sign changing solutions of nonlinear elliptic problems, Mathematische Zeitschrift, 233(2000), 655-677.

[2] E. Cances, H. Galicher and M. Lewin, Computing electronic structures: a new multiconfiguration approach for excited states, Research Report No. 5289(2004), Institut National De Recherche En Informatique Et En Automatique.

[3] S.-M. Chang, C.-S. Lin, T.-C. Lin and W.-W. Lin, Segregated nodal domains of two dimensional multispecies Bose-Einstein condensates, Physica D, 196(2004), 341-461.

[4] X. Chen and J. Zhou, Estimates of Morse indices in numerical computations via a local minorthogonal method, preprint, 2006.

[5] R.Y. Chiao, E. Garmire and C.H. Townes, Self-trapping of optical beams, Phys. Rev. Lett. 13(1964), 479482.

[6] Y.S. Choi and P.J. McKenna, A mountain pass method for the numerical solution of semilinear elliptic problems, Nonlinear Analysis, 20(1993), 417-437.

[7] A.S. Desyatnikov, D. Neshev, E.A. Ostrovskaya and Y.S. Kivshar, Multipole composite spatial solitons: theory and experiment, J. Opt. Soc. Am. B, 19(2002), 586-595.

[8] A.S. Desyatnikov, D. Neshev, E.A. Ostrovskaya and Y.S. Kivshar, Multipole spatial vector solitons, Optics Letters, 26(2001), 435-437. 
[9] Z. Ding, D. Costa and G. Chen, A high linking method for sign changing solutions for semilinear elliptic equations, Nonlinear Analysis, 38(1999), 151-172.

[10] J.J. Garcia-Ripoll and V.M. Perez-Garcia, Stable and unstable vortices in multicomponent Bose-Einstein condensates, Phy. Rev. Lett., 84(2000), 4265-4267.

[11] J.J. Garcia-Ripoll, V.M. Perez-Garcia, E.A. Ostrovskaya and Y.S. Kivshar, Dipole-mode vector solitons, Phy. Rev. Lett., 85(2000), 82-85.

[12] K. Kasamatsu, M. Tsubota and M. Ueda, Vortices in multicomponent Bose-Einstein condensates, Int. J. Mod. Phys. B, 19(2005), 1835-1904.

[13] W. Krolikowski, G. McCarthy and Y.S. Kivshar, Scattering of dipole-mode vector solitons: theory and experiment, Physical Review E 68, 016612 (2003).

[14] Y.S. Kivshar and G.I. Stegeman, Spatial optical solitons, Optics \& Photonics News, Feb. 2002, 59-63.

[15] Y. Li and J. Zhou, A minimax method for finding multiple critical points and its application to semilinear PDE, SIAM J. Sci. Comput., 23(2001), 840-865.

[16] Y. Li and J. Zhou, Convergence results of a minimax method for finding multiple critical points, SIAM J. Sci. Comput., 24(2002), 840-865.

[17] K.W. Madison, F. Chevy, W. Wohlleben and J. Dalibard, Vortex formation in a stirred BoseEinstein condensate, Phys. Rev. Lett., 84(2000), 806-809.

[18] Z.H. Musslimani, M. Segev, D.N. Christodoulides and M. Soljacic, Composite multihump vector solitons carrying topological charge, Phy. Rev. Lett., 84(2000), 1164-1167.

[19] W.-M. Ni, Some Aspects of Semilinear Elliptic Equations, Dept. of Math., National Tsing Hua Univ., Hsinchu, Taiwan, Rep. of China, 1987.

[20] E.A. Ostrovskaya, Y.S. Kivshar, D.V. Skryabin and W.J. Firth, Stability of multihump optical solitons, Phys. Rev. Lett., 83(1999), 296-299.

[21] A. Pomponio, Asymptotically linear cooperative elliptic system: existence and multiplicity, Nonlinear Analysis, 52(2003), 989-1003.

[22] Ch. Ruegg, N. Cavadini, A. Furrer, H.-U. Gudel, K. Kramer, H. Mutka, A. Wildes, K. Habicht and P. Vorderwisch, Bose-Einstein condensation of the triplet states in the magnetic insulator TlCuCl3, Nature, 423(2003), 62-65.

[23] J. R. Salgueiro, Y. S. Kivshar, D. E. Pelinovsky, V. Simon and H. Michinel, Spatial vector solitons in nonlinear photonic crystal fibers, Studies in Appl. Math., 115(2005), 157-171.

[24] J. Schulz, M. Tchaplyguine, T. Rander, O. Bjorneholm, S. Svensson, R. Sankari, S. 
Heinasmaki, H. Aksela, S. Aksela and E. Kukk, Shakedown in core photoelectron spectra from aligned laser-excited Na atoms, Physical Review A 72, 010702 (2005)

[25] J. Schulz, M. Tchaplyguine, T. Rander, H. Bergersen, A. Lindblad, G. Ohrwall, S. Svensson, S. Heinasmaki, R. Sankari, S. Osmekhin, S. Aksela and H. Aksela, Final state selection in the $4 \mathrm{p}$ photoemission of Rb by combining laser spectroscopy with soft-x-ray photoionization, Physical Review A 72, 032718 (2005).

[26] J. Smoller, Shock Waves and Reaction-diffusion Equations, Springer-Verlag, New York, 1982.

[27] S.I. Themelis, P. Lambropoulos and F.J. Wuilleumier, Laser-induced transitions between core excited states of Na, J. Phys. B, 38(2005), 2119-2132.

[28] Z.-Q. Wang and J. Zhou, A local minimax-newton method for finding critical points with symmetries, SIAM J. Num. Anal., 42(2004), 1745-1759.

[29] Z.-Q. Wang and J. Zhou, An efficient and stable method for computing multiple saddle points with symmetries, SIAM J. Num. Anal., 43(2005), 891-907.

[30] M. Willem, Minimax Theorems, Progress in Nonlinear Differential Equations and Their Applications, Vol. 24, Birkhäuser, Boston, 1996.

[31] J. Yang and D.E. Pelinovsky, Stable vortex and dipole vector solitons in a saturable nonlinear medium, Physical Review E 67, 016608 (2003).

[32] X. Yao and J. Zhou, A minimax method for finding multiple critical points in Banach spaces and its application to quasilinear elliptic PDE, SIAM J. Sci. Comp., 26(2005), 1796-1809.

[33] J. Zhang, Existence results for the positive solutions of nonlinear elliptic systems, Applied Mathematics and Computation, 153(2004), 833-842.

[34] P. Zhao and X. Wang, The existence of positive solution of elliptic system by a linking theorem on product space, Nonlinear Analysis, 56(2004), 227-240.

[35] J. Zhou, A local min-orthogonal method for finding multiple saddle points, J. of Mathematical Analysis and Applications, 291(2004), 66-81.

[36] J. Zhou, Instability analysis of saddle points by a local minimax method, Math. Comput., 74(2005), 1391-1411.

[37] W.-M. Zou, Multiple solutions for asymptotically linear elliptic systems, J. of Mathematical Analysis and Applications 255(2001), 213-229. 